\title{
Observable signatures of dust evolution mechanisms which shape the planet forming regions
}

\author{
Olja Panić $^{1}$, Tilman Birnstiel ${ }^{2}$, Ruud Visser $^{3}$ and Elco van Kampen ${ }^{1}$ \\ ${ }^{1}$ European Southern Observatory, \\ Karl-Schwarzschild Strasse 2, 85748, Garching bei München, Germany \\ email: opanic@eso.org \\ ${ }^{2}$ Max-Planck-Institut für Astronomie, Königstuhl 17, 69117 Heidelberg, Germany \\ ${ }^{3}$ Leiden Observatory, Leiden University, PO Box 9513, 2300 RA Leiden, The Netherlands
}

\begin{abstract}
Overdensity of dust with respect to the gas in the planet forming regions is a crucial prerequisite to form larger bodies and eventually planets. We use a state-of-the-art code to simulate dust evolution processes in gas-rich circumstellar discs, including the viscous gas evolution. We find significant deviations of the radial distribution of dust from that of the gas as early as $1-2 \mathrm{Myr}$. These deviations are closely related to the efficiency of grain growth. Apparent discrepancies between dust and gas distributions are suggested by the current millimetre interferometer observations, and ALMA will allow us tointerpret any such discrepancies in the context of dust evolution.
\end{abstract}

Keywords. stars: pre-main-sequence, circumstellar matter, planetary systems: protoplanetary disks, planets and satellites: formation

\section{Model description}

We use the dust evolution code based on Brauer et al. (2008) and Birnstiel et al. (2010). The dust growth, fragmentation, radial drift and radial mixing processes are treated in the context of a viscously evolving, irradiated gas disc. We focus on two sets of models, their initial conditions differing only by the assumed limiting relative velocity, $v_{f}$, at which the dust grains fragment rather than coagulate upon impact. For low values of $v_{f}$, the dust fragments efficiently and remains rather small in size during the evolution of the disc, while for high values of $v_{f}$ the dust grows to larger sizes. Our two models have $v_{f}=1 \mathrm{~m} / \mathrm{s}$ (Model A) and $10 \mathrm{~m} / \mathrm{s}$ (Model B). The main model parameters are the disc radial size set to $250 \mathrm{AU}$, gas mass of $0.1 M_{\odot}$ and dust to gas mass ratio of $1: 100$. These parameters are fixed at time $=0$ and vary during the calculation due to aforementioned processes. A more detailed description of the model parameters can be found in Birnstiel et al. 2010.

The dust size is an important factor in assessing the effects of radial drift of dust in discs. The choice of fragmentation velocity value, $v_{f}$, directly influences the dust size and drift efficiency in our models. Our study examines the consequences of dust evolution, and in particular the radial drift, on the disc structure as a whole, and not only in the planet forming region. The motivation for this is the prospect of directly probing the outer disc regions at high sensitivity with the Atacama Large Millimeter/submillimeter Array in near future. 

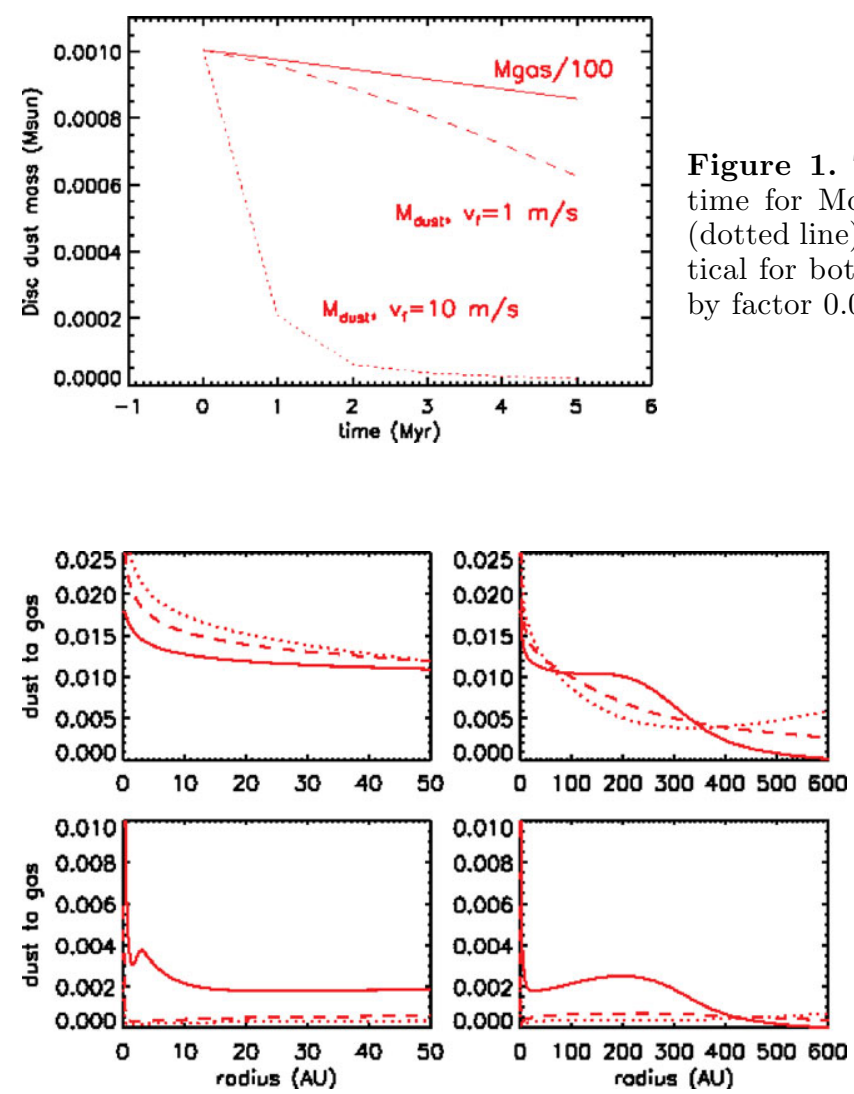

Figure 2. Upper panels: The dust to gas mass ratio in Model A at 1 Myr (solid lines), 2 Myr (dashed lines) and 3 Myr (dotted lines). Lower panels: Same as in upper panels, for Model B.

\section{Results: Dust to gas mass ratio}

The influence that dust growth and drift have on disc dust mass is most pronounced in Model B (see Fig. 1). At time of 1 Myr the disc dust mass has decreased by $80 \%$ due to efficient radial drift and consequent accretion onto the star. Model A exhibits a dust mass decrease but at a notably slower rate of only $20 \%$ over $5 \mathrm{Myr}$. The gas evolves independently of the dust in our models, including only accretion and viscous evolution. The disc gas mass decreases more gradually than the dust (shown scaled by factor 0.01 in Fig. 1 for comparison).

The overall dust to gas mass ratio of the disc in both our models decreases over time. The most affected are the disc outer regions where most mass resides and where the optimal dust size for drift is small. Figure 2 shows the evolution on the dust to gas mass ratio in Models A (upper panels) and B (lower panels). In Model A the dust is depleted from the outer disc and builds up in the planet forming regions, because the dust remains smaller than the optimal drift size corresponding to the physical properties in these regions. In Model B, the dust grows efficiently and drifts throughout the disc, causing a decrease of the dust to gas mass ratio at all radii.

An increase in the amount of solids in the inner disc is a necessary pre-requisite for planet formation. Our modelling suggests that local processes, such as clumping due to streaming instabilities, are necessary to allow dust to grow to large sizes whilst retaining it in the planet forming regions. 


\section{References}

Birnstiel, T., Dullemond, C. P., \& Brauer, F. 2010, A\& $A$, 513, A79

Brauer, F., Dullemond, C. P., \& Henning, Th. 2008, A\&GA, 480, 859 